\title{
Elevated level of anterior gradient-2 in pancreatic juice from patients with pre-malignant pancreatic neoplasia
}

\author{
Ru Chen*1, Sheng Pan², Xiaobo Duan³, Brad H Nelson³, Rob A Sahota3, Sarah de Rham³, Richard A Kozarek4, \\ Martin McIntosh ${ }^{5}$ and Teresa A Brentnall ${ }^{1}$
}

\begin{abstract}
Background: Pancreatic intraepithelial neoplasias (PanINs) are precursors of malignant pancreatic cancer, an ideal stage for early cancer detection. We applied quantitative proteomics to identify aberrantly elevated proteins in pancreatic juice samples derived from patients with PanIN3.

Results: Twenty proteins were found elevated in all three PanIN juices by at least two-fold. Among these proteins, anterior gradient-2 (AGR2) was found to be 2-10 fold elevated in PanIN3 juice samples analyzed by quantitative proteomics. An ELISA assay was developed to evaluate AGR2 levels in 51 pancreatic juice samples and 23 serum samples from patients with pancreatic cancer, pre-malignant lesions (including PanIN3, PanIN2, Intraductal Papillary Mucinous Neoplasms (IPMNs)) and benign disease controls (including chronic pancreatitis). AGR2 levels in the pancreatic juice samples were found significantly elevated in patients with pre-malignant conditions (PanINs and IPMNs) as well as pancreatic cancer compared to control samples ( $p \leq 0.03)$. By ROC analysis, the AGR2 ELISA achieved $67 \%$ sensitivity at $90 \%$ specificity in predicting PanIN3 juice samples from the benign disease controls.

Conclusions: These results suggest that elevation of AGR2 levels in pancreatic juice occurs in early pancreatic cancer progression and could be further investigated as a potential candidate juice biomarker for early detection of pancreatic cancer.
\end{abstract}

\section{Introduction}

Pancreatic cancer is the fourth leading cause of cancer death in the United States [1]. The poor prognosis could potentially be improved by development of biomarkers that could be used for early detection. Pancreatic intraepithelial neoplasia or PanIN, represents the precursor lesion for pancreatic ductal adenocarcinoma and is graded 1-3, with PanIN3 representing the stage just before cancer. Advanced PanIN lesions, e.g. PanIN3, would be an ideal stage to diagnose patients, as this represents a time point when intervention and cure is possible.

Pancreatic juice is a proximal body fluid and represents an opportune specimen for identifying biomarkers of pancreatic cancer. Cancer cells are preferentially shed

* Correspondence: ruc@medicine.washington.edu

1 Gl Division/Department of Medicine, University of Washington, Seattle, WA 98195 USA

Full list of author information is available at the end of the article into the ductal lumen, making juice a rich source of cancer associated markers. Previous studies have been conducted to investigate telomerase, microRNA, methylation, DNA mutation, and aberrant proteins as potential biomarkers in pancreatic juice from patients with pancreatic cancer or IPMNs (intraductal papillary mucinous neoplasms) [2-10]. However, to our knowledge, there has not been any study to evaluate pancreatic juice samples from patients with PanIN3 lesions.

In this study, we first applied mass spectrometry based quantitative proteomics to globally profile pancreatic juice samples from patients with histologically confirmed PanIN3 (referred as PanIN3 juice) to identify proteins with differential expression level in comparison to juice from benign disease controls. Among the differential proteins revealed, AGR2 was elevated in all PanIN3 juice samples analyzed. A recent study showed that AGR2 was highly expressed in the tissue of both PanIN lesions and 
pancreatic cancer [11]. The same study also demonstrated that AGR2 was secreted into culture media by pancreatic cancer cells. To further investigate AGR2 levels in pancreatic juice samples and sera, we developed AGR2-specific monoclonal antibodies (mAbs) and an ELISA to quantitatively detect AGR2 in pancreatic juice and blood samples. We compared the AGR2 levels in samples (juice and serum) from controls (including benign diseases and chronic pancreatitis), patients with pre-malignant lesions (PanIN2, PanIN3 and IMPNs), and patients with pancreatic cancer. Statistical analyses were used to determine significance between each sample group, and the sensitivity and specificity of AGR2 in separating cases from controls.

\section{Materials and methods}

\section{Specimens}

The specimens were collected in accordance with approved Human Subject's guidelines at the University of Washington and Virginia Mason Medical Center. The pancreatic juice samples were collected during endoscopic retrograde cholangiopancreaticography (ERCP) and immediately stored at $-80^{\circ} \mathrm{C}$. The control juice samples were from: 1) 7 cancer-free patients who were undergoing evaluation of Sphincter of Oddi dysfunction; 2) 11 patients who have benign pancreatic diseases, such as chronic pancreatitis. The PanIN2 juice samples were obtained from 6 patients who had histologically proven PanIN2 but without pancreatic cancer. The PanIN3 juice samples were obtained from 9 patients who had histologically proven PanIN3 but without pancreatic cancer. These patients with PanIN diagnoses had complete pancreas resection, and no cancer found in the pancreas[12]. The IPMN cases were benign to borderline without evidence of malignancy. The pancreatic cancer juice samples were from 8 patients who had pancreatic ductal adenocarcinoma including stages 2 to 4 . The diagnosis of disease was made histologically or, in the case of the controls, by imaging in combination with supporting laboratory values. The patient demographics are presented in Table 1. Serum samples were obtained in red top tubes and processed within 4 hours using a uniform protocol. Once processed the serum was stored at $-80^{\circ} \mathrm{C}$ and no more than 2 freeze thaw cycles were allowed for a specimen used in the ELISA studies. Six sera from patients with PanIN2-3 and 9 sera from pancreatic cancer patients were included in this study for AGR2 serum ELISA testing. The 9 healthy control sera were purchased from Innovative Research (Southfield, MI).

\section{Quantitative proteomics}

The 4-plex iTRAQ (isobaric tags for relative and absolute quantification) method [13] was applied in combination with tandem mass spectrometry for quantitative profiling
Table 1: Patient demographics in pancreatic juice

\begin{tabular}{|c|c|c|c|}
\hline & $\begin{array}{l}\text { Benign } \\
(N=18)\end{array}$ & $\begin{array}{l}\text { Premalignant } \\
\quad(\mathrm{N}=\mathbf{2 5})\end{array}$ & $\begin{array}{c}\text { Pancreatic } \\
\text { cancer } \\
(\mathrm{N}=\mathbf{8})\end{array}$ \\
\hline \multicolumn{4}{|l|}{ Age } \\
\hline Mean & 47.75 & 54.88 & 69.5 \\
\hline Range & $18-73$ & $25-79$ & $46-77$ \\
\hline \multicolumn{4}{|l|}{ Sex } \\
\hline Male & 4 & 12 & 3 \\
\hline Female & 14 & 13 & 3 \\
\hline Unknown & 0 & 0 & 2 \\
\hline \multicolumn{4}{|l|}{ Benign } \\
\hline Sphincter of Oddi & 7 & & \\
\hline Chronic pancreatitis & 11 & & \\
\hline \multicolumn{4}{|l|}{ Premalignant } \\
\hline PanIN2 & & 6 & \\
\hline PanIN3 & & 9 & \\
\hline IPMN & & 10 & \\
\hline \multicolumn{4}{|l|}{ Malignant } \\
\hline stage 2 & & & 1 \\
\hline stage 3 & & & 3 \\
\hline stage 4 & & & 2 \\
\hline stage undetermined & & & 2 \\
\hline
\end{tabular}

of the pancreatic juice proteome from PanIN3 cases and normal control. The control sample was generated by pooling equal volume of 5 pancreatic juice samples from patients with benign disease. Three hundred microliters of pancreatic juice samples from pooled normal juice, and three separate PanIN3 cases (a, b, and c) were subjected to iTRAQ labeling followed by mass spectrometer analysis using similar methods as previously described[14]. The MS/MS data were analyzed and processed using Trans-Proteomic Pipeline (TPP). All of the MS/MS spectra were searched against IPI human protein database (v3.38) using the SEQUEST algorithm[15]. The database search results were validated using the PeptideProphet [16] and ProteinProphet [17]. ProteinProphet probability score $>0.9$ was used as the cut-off value for protein identification to ensure that the false positive rate (error rate) for protein identification was $<0.9 \%$. iTRAQ quantification on peptide and protein abundance was achieved using LIBRA program[18]. More information about Trans-Proteomic Pipeline, PeptideProphet, Protein- 
Prophet, LIBRA and other programs can be obtained from the Seattle Proteome Center http://tools.proteomecenter.org.

\section{Production of recombinant AGR2 protein and monoclonal antibodies}

A cDNA encoding AGR2 protein (21-175 aa) was reverse transcribed from the OVCAR-3 cell line (ATCC, Manassas, VA) and verified by DNA sequence analysis. Recombinant AGR2 protein (rAGR2) was expressed in Escherichia coli with a GST tag and purified on GSTrap columns (GE Healthcare, Fairfield, CT) using the AKTA Prime (GE Healthcare) purification system. Full length, GST-tagged recombinant AGR3 (rAGR3) protein was purchased (Novus Biologicals, Littleton, CO). rAGR2 was used to immunize BALB/c mice, and spleens from mice with high-titer antibody responses to AGR2 were used to develop mAbs using standard hybridoma technology. Hybridomas were cloned by limiting dilution, and the supernatants from each hybridoma were screened against the GST-tagged immunogen and, as negative controls, other irrelevant GST-tagged recombinant proteins. mAb affinities for AGR2 were evaluated with a Biacore X100 instrument (GE Healthcare).

\section{AGR2 immunoprecipitation and mass spectrometric identification}

To ensure that the newly developed AGR2.43 mAb was binding specifically to AGR2 protein, we performed an immunoprecipitation using this antibody and then sequenced the immunoprecipitated product using mass spectrometry. OV90 cell line lysate was used as the source of AGR2. OV90 cell line lysate (1 mg in $1 \mathrm{ml})$ was pre-cleared by incubation with $50 \mathrm{ul}$ of Protein G resin (Pierce, Rockford, IL) for 30 minutes at $4^{\circ} \mathrm{C}$ with shaking. Protein $\mathrm{G}$ was pelleted by centrifugation and discarded. Five microgram of AGR2.43 antibody was coupled to 50 ul of Protein $\mathrm{G}$ resin by incubation for 1 hour at $4^{\circ} \mathrm{C}$ with shaking. The AGR2 antibody-Protein G complex was centrifuged, washed in PBS, centrifuged again and added to the pre-cleared OV90 cell line lysate and incubated for 2 hours at $4^{\circ} \mathrm{C}$ with shaking. The complex was washed and centrifuged 3 times and resuspended in PBS. The complex was dissociated from protein $\mathrm{G}$ by incubation with $50 \mathrm{ul}$ of $100 \mathrm{mM}$ glycine at $\mathrm{pH}$ 2.5. Following centrifugation and removal of the protein $\mathrm{G}$ pellet, the remaining sample was neutralized by addition of $1 \mathrm{M}$ Tris- $\mathrm{HCl}$ pH 8.5 and further prepared as described below for Mass Spectrometry analysis.

The AGR2 immunoprecipitated pull-down product was buffer exchanged with $50 \mathrm{mM}$ sodium bicarbonate, reduced with $20 \mathrm{mM}$ dithiothreitol at $37^{\circ} \mathrm{C}$ for 1 hour and incubated with $20 \mathrm{mM}$ iodoacetamide in the dark for 30 minutes to block the cysteine groups. The proteins were then digested with trypsin (Promega, Madison, WI) at a 1:50 ratio for 18 hours at $37^{\circ} \mathrm{C}$. The obtained peptides were purified with a C18 column (The Nest Group, Southborough, MA) and subjected to LC MS/MS analysis using a linear ion trap mass spectrometer (LTQ, ThermoFinnigan, San Jose, CA). The resulting data was searched against IPI human protein database V. 3.38 for peptide and protein identification using SEQUEST algorithm. A 1\% false positive rate based on ProteinProphet was used as a cut-off value for protein identification.

\section{Western Blotting}

Cells from the human pancreatic cancer cell lines CFPAC-1 and MiaPaca were harvested and treated with lysis buffer (PBS; 0.05\% TritonX-100) including protease inhibitor cocktail (Roche, Mannheim, Germany). Following sonication and centrifugation, cell lysate supernatants were quantified using a BCA quantification kit (Sigma, Oakville, ON, Canada) and stored at $-80^{\circ} \mathrm{C}$. Ten micrograms of cell line lysate and 10 nanograms of rAGR2 or rAGR3 were separated using NuPAGE Novex $4 \%-12 \%$ Bis-Tris gels at $200 \mathrm{~V}$ for 40 minutes and transferred to nitrocellulose using the XCell SureLock Mini-Cell (Invitrogen) at $30 \mathrm{~V}$ for 60 minutes. Membranes were blocked overnight in blocking buffer (50 mM Tris; $150 \mathrm{mM} \mathrm{NaCl}$; $5 \%$ skim milk powder). Membranes were then incubated with $2 \mu \mathrm{g} / \mathrm{ml}$ AGR2.43 in blocking buffer with $0.1 \%$ Tween-20 for 4 hours, washed three times in TBST (50 $\mathrm{mM}$ Tris; $150 \mathrm{mM} \mathrm{NaCl}$; $0.1 \%$ Tween-20), incubated for 1 hour in Goat anti-mouse IR-800 (LI-COR, Lincoln, NE), washed again and visualized with the Odyssey infrared imager (LI-COR). As a loading control, the same membrane was further probed with an anti-GAPDH antibody (STEMCELL Technologies, Vancouver, Canada), reprobed with Goat anti-mouse IR-800 and imaged on the Odyssey infrared imager.

\section{AGR2 ELISA}

To develop the ELISA, antibody pairs were identified using hybridoma supernatants from AGR2 specific hybridoma clones with a Biacore X100 instrument (GE Healthcare). Mouse IgGs were purified using a protein $G$ column (GE Healthcare) and labeled with biotin using a kit (Thermo Scientific). The mAb pair (mAbs AGR2.43 and AGR2.44) that showed the best sensitivity to specifically detect rAGR2 in both $1 \times$ Reagent Diluent (RD; R\&D Systems, Minneapolis, MN) and in RD supplemented with $10 \%$ normal human serum (NHS) was selected. A sandwich ELISA was established using mAb AGR2.43 as capture, mAb AGR2.44 as detector and Superblock (Thermo Scientific) as blocking buffer. The ELISA was further optimized by determining the optimal incubation time and the concentration of the capture and detector antibody, the streptavidin-alkaline phosphatase conjugate 
(Applied Biosystems Inc, Foster City, CA) and the chemiluminescent $\mathrm{CSPD}^{\circ}$ Substrate to achieve the highest sensitivity.

To assess the performance characteristics of the AGR2 ELISA, we tested serially diluted rAGR2 protein (from 40 $\mathrm{ng} / \mathrm{ml}$ to $0.055 \mathrm{ng} / \mathrm{ml}$ in RD or RD $10 \% \mathrm{NHS}$ ) and diluted human serum and cell line lysates (diluted 1/10 in RD) in triplicate in three independent ELISA experiments. For each experiment, a five-parameter log-logistic (5PL) standard curve was generated using EnVision software version 1.12 and GraphPad Prism, and used to backcalculate the concentration of the rAGR2 standards and AGR2 levels in the human serum samples and cell line lysates. The means, signal-to-noise $(\mathrm{S}: \mathrm{N})$ ratios, intraand inter-assay standard deviations (SD), and percent coefficient of variances $(\% \mathrm{CV})$ were calculated.

AGR2 ELISAs were performed by researchers who were blinded to the case/control status of specimens. Costar white high binding 96 well plates (Corning, Corning, NY) were coated with $100 \mu \mathrm{l} /$ well of $1.25 \mu \mathrm{g} / \mathrm{ml}$ purified mAb AGR2.43 in $0.1 \mathrm{M}$ carbonate buffer $(33.5 \mathrm{mM}$ $\mathrm{Na}_{2} \mathrm{CO}_{3}, 0.1 \mathrm{M} \mathrm{NaHCO}, \mathrm{pH}$ 9.6) and incubated overnight at $4^{\circ} \mathrm{C}$. Plates were blocked with $200 \mu \mathrm{l} /$ well of Superblock (Pierce, Rockford, IL) and incubated at room temperature (RT) for 2.5 hours. Plates were washed with a protocol including six wash steps in TBST using a Skanwasher plate washer (Molecular Devices, Union City, CA). Patient serum, control serum or pancreatic juice was diluted 1:10 in RD and incubated for 2 hours at RT on a shaker. All samples and controls were assayed in duplicate. Plates were washed and incubated with $100 \mu \mathrm{l}$ per well of $0.25 \mu \mathrm{g} / \mathrm{ml}$ biotinylated mAb AGR2.44 in TBST for 2 hours at RT with shaking. Plates were washed and incubated with $100 \mu \mathrm{l}$ per well streptavidin-alkaline phosphatase conjugate at 1:2500 in TBST for 1 hour on a shaker at RT. After washing, the plates were incubated with $100 \mu \mathrm{l} /$ well of $0.4 \mathrm{mM}$ chemiluminescent CSPD ${ }^{\circ}$ Substrate with Emerald-II ${ }^{\mathrm{IM}}$ Enhancer (Applied Biosystems) at RT for 20 min in the dark, read on an EnVision multilabel plate reader (PerkinElmer, Waltham, Massachusetts), and analyzed using Envision software 1.12.

\section{Immunohistochemistry (IHC)}

Pancreatic tissues from 2 PanINs, 2 pancreatic cancers and 2 cancer free controls were analyzed by IHC. Pancreatic tissue blocks were sectioned at $5 \mu \mathrm{m}$ onto slides and incubated for 30 minutes at $58^{\circ} \mathrm{C}$. The slides were deparaffinized before placing in a Ventana Discovery XT autostainer (Ventana, Tucson, AZ) for immunohistochemical staining. Antigen retrieval was performed with a Ventana's standard CC1 protocol. The slides were first incubated with mAb AGR2.43 for 60 minutes, and the appropriate cross-adsorbed, biotinylated secondary antibody (Jackson Immunoresearch, West Grove, PA) was applied for 32 minutes. Bound antibodies were detected using the DABMap kit (Ventana), counterstained with hematoxylin (Ventana), and coverslipped manually with Cytoseal-60 (Richard Allan, Kalamazoo, MI). Two slides from each tissue block were stained in separate experiments. The specificity of mAb AGR2.43 was confirmed by blocking experiments. Specifically, incubation of mAb AGR2.43 with rAGR2, but not rAGR3, resulted in loss of signal in subsequent AGR2 IHC staining.

\section{Statistical analysis}

For the purpose of statistical analysis, variables regarding patient diagnostics were grouped in the following manner: control (including all benign diseases from patients undergoing a work-up for Sphincter of Oddi dysfunction, and patients with pancreatitis), premalignant lesions (including PanIN2, PanIN3, and IPMNs), and malignant cancer groups (Table 1). Statistical analyses were performed using GraphPad Prism (La Jolla, CA). Differences in the AGR2 levels between groups of patients were tested for statistical significance using the Mann-Whitney test in case of two groups and the Kruskal Wallis test in case of more than two groups, respectively. Empirical receiver operating characteristic (ROC) curves were used to determine the sensitivity and specificity of AGR2 in separating cases from controls. ROC curve shows the trade-off between sensitivity and specificity as the threshold for defining a positive test is varied. Generalized linear models were used to test for association between the AGR2 values in serum and pancreatic juice. Statistical significance was defined as $P<0.05$.

\section{Results}

Identification of elevated proteins in pancreatic juice samples from patients with PanIN3 by quantitative proteomics

Quantitative proteomics analysis using stable isotope labeling (iTRAQ) and tandem mass spectrometry was applied to identify proteins abnormally elevated in the pancreatic juice samples from PanIN3 patients. A pool of 5 control pancreatic juice samples was used as a control for quantitative comparison. We identified twenty proteins displaying elevated levels by at least two-fold in all three PanIN3 juices when compared to normal control juice (Table 2). Among these elevated proteins, anterior gradient-2 (AGR2) was elevated by $10.47 \pm 0.01 ; 3.96 \pm$ 0.03 ; and $2.04 \pm 0.01$ folds in the three PanIN3 pancreatic juice samples respectively, comparing to the pooled control sample (Figure 1). A recent study using IHC showed that AGR2 was highly expressed in both PanINs and pancreatic cancer tissues and is secreted into the culture media of pancreatic cancer cell lines [11]. This, together 
A. HLSPDGQYVPR, $\mathrm{MH}+1413.4857, \mathrm{~m} / \mathrm{z} 471.8334$

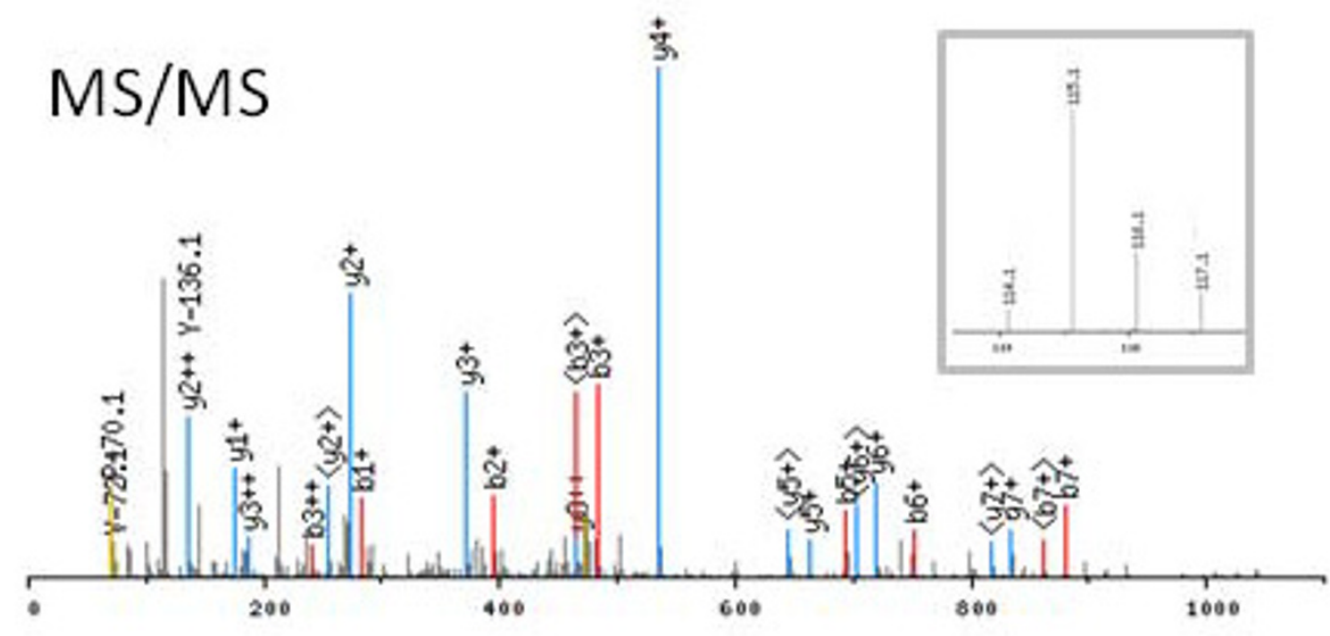

B. LYAYEPADTALLLDNMK, $\mathrm{MH}+2229.2471, \mathrm{~m} / \mathrm{z} 743.7539$

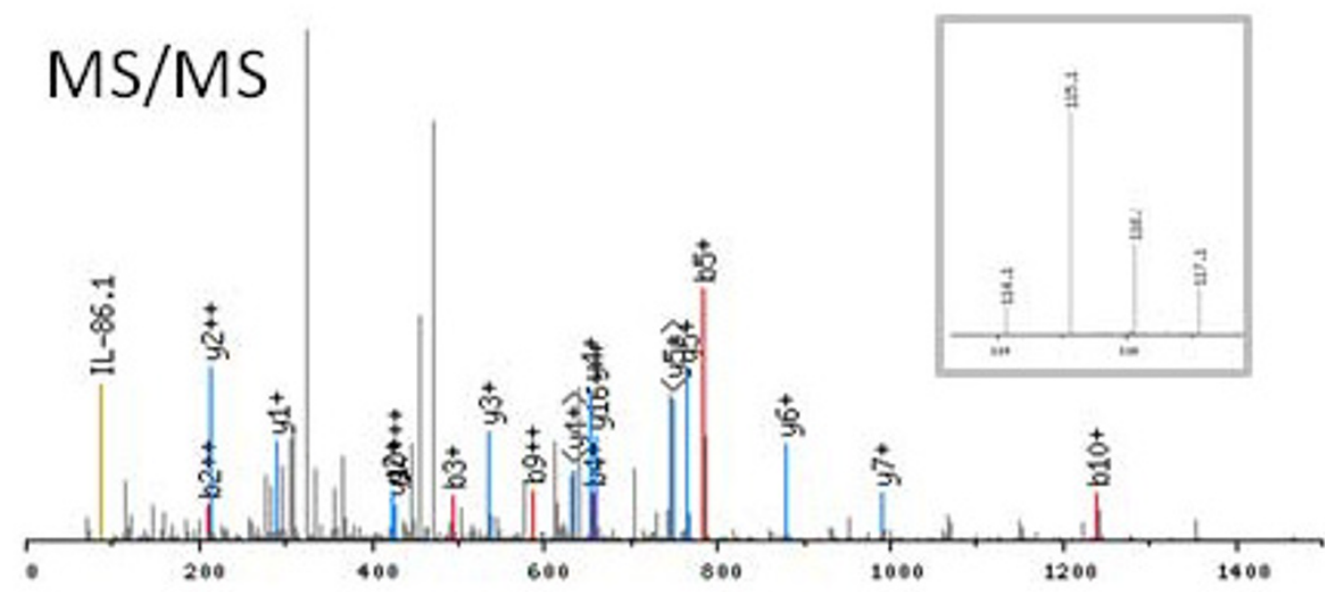

Figure 1 Identification and quantification of AGR2 in pancreatic juice by iTRAQ labeling and tandem mass spectrometry. Two unique peptides (HLSPDGQYVPR and LYAYEPADTALLLDNMK) from AGR2 were identified; the MS/MS spectra are presented in panel A and panel B respectively. The inserts are the ITRAQ reporting peaks at 114,115, 116 and 117 representing relative intensity of the 4 samples compared: 114, pooled normal control juices; 115-117 were from PanIN case $a$, case $b$ and case $c$ respectively.

with our results, prompted us to further investigated AGR2 for its utility as a biomarker for pancreatic cancer.

\section{Production and evaluation of AGR2 antibodies}

We generated multiple hybridoma clones producing antibodies specific for AGR2. Purified mAbs from these hybridomas were then assessed for their specificity to AGR2 using Western blotting, mass spectrometry, ELISA and IHC. By Western blot, mAb AGR2.43 detected the 45
kDa GST-tagged rAGR2, but not GST-tagged rAGR3 protein (Figure 2). This antibody also detected a $20 \mathrm{kDa}$ protein corresponding to the predicted molecular weight of native AGR2 in the cell lysate of CFPAC-1, which expresses a high level of AGR2, but not in the pancreatic cancer cell line MiaPaca, which expresses a low level of AGR2 (Figure 2), nor in the normal pancreatic ductal cell line HPDE (data not shown). mAb AGR2.44 likely detects a discontinuous epitope as the antibody failed to detect 
Table 2: Abnormally elevated proteins identified in PanIN3 juices by quantitative proteomics (by at least two-fold change)

\begin{tabular}{|c|c|c|c|c|c|c|c|}
\hline gene symbol & protein description & $\begin{array}{c}\text { ratio PanIN3 } \\
\text { case a vs normal }\end{array}$ & PanIN3 case a SEM & $\begin{array}{l}\text { ratio PanIN3 case } \\
\text { b vs normal }\end{array}$ & PanIN3 case b SEM & $\begin{array}{l}\text { ratio PanIN3 case } \\
\text { c vs normal }\end{array}$ & PanIN3 case c SEM \\
\hline AGR2 & Anterior gradient-2 & 10.47 & 0.03 & 3.96 & 0.01 & 2.04 & 0.01 \\
\hline HIST1H2B & Histone $\mathrm{H} 2 \mathrm{~B}$ & 34.78 & 0.04 & 8.53 & 0.03 & 10.01 & 0.01 \\
\hline LYZ & Lysozyme C & 27.56 & 0.02 & 3.70 & 0.01 & 2.05 & 0.01 \\
\hline MUC5AC & Mucin-5AC & 31.06 & 0.01 & 3.83 & 0.00 & 4.54 & 0.00 \\
\hline CA2 & Carbonic anhydrase 2 & 9.58 & 0.02 & 3.18 & 0.01 & 1.97 & 0.01 \\
\hline KLK1 & Kallikrein-1 & 3.25 & 0.06 & 3.12 & 0.08 & 11.52 & 0.02 \\
\hline ANXA5 & Annexin A5 & 21.46 & 0.01 & 4.43 & 0.01 & 9.70 & 0.01 \\
\hline $\mathrm{HIST} 2 \mathrm{H} 4 \mathrm{~A}$ & Histone $\mathrm{H} 4$ & 41.77 & 0.01 & 9.82 & 0.01 & 11.88 & 0.01 \\
\hline ANXA4 & Annexin A4 & 18.39 & 0.02 & 4.28 & 0.01 & 5.11 & 0.01 \\
\hline АСТВ & Actin, cytoplasmic 1,2 & 17.98 & 0.01 & 6.13 & 0.01 & 4.41 & 0.01 \\
\hline SERPINA1 & Alpha-1-antitrypsin & 11.03 & 0.04 & 4.30 & 0.02 & 1.99 & 0.02 \\
\hline KRT8 & Keratin, type II cytoskeletal 8 & 19.25 & 0.02 & 4.26 & 0.01 & 3.72 & 0.01 \\
\hline PRSS1 & PRSS1 protein & 4.56 & 0.01 & 15.35 & 0.02 & 30.46 & 0.03 \\
\hline YWHAE & 14-3-3 protein epsilon & 15.60 & 0.02 & 4.48 & 0.01 & 2.42 & 0.01 \\
\hline $\begin{array}{l}\text { PPIA;LOC654188;PPIA } \\
\text { L3 }\end{array}$ & $\begin{array}{l}\text { Peptidyl-prolyl cis-trans } \\
\text { isomerase A }\end{array}$ & 13.93 & 0.02 & 5.05 & 0.02 & 4.04 & 0.01 \\
\hline PSIP1 & $\begin{array}{l}\text { PC4 and SFRS1-interacting } \\
\text { protein }\end{array}$ & 2.68 & 0.01 & 17.91 & 0.00 & 12.16 & 0.01 \\
\hline AKR1B10 & $\begin{array}{l}\text { Aldo-keto reductase family } 1 \\
\text { member B10 }\end{array}$ & 14.60 & 0.01 & 8.32 & 0.01 & 5.01 & 0.00 \\
\hline CLIC1 & $\begin{array}{l}\text { Chloride intracellular channel } \\
\text { protein } 1\end{array}$ & 5.84 & 0.04 & 3.22 & 0.04 & 4.08 & 0.04 \\
\hline C2 & Complement C2 & 4.94 & 0.01 & 24.53 & 0.02 & 17.69 & 0.03 \\
\hline HNRNPA2B1 & $\begin{array}{l}\text { Heterogeneous nuclear } \\
\text { ribonucleoproteins }\end{array}$ & 7.13 & 0.04 & 2.64 & 0.01 & 2.51 & 0.04 \\
\hline
\end{tabular}




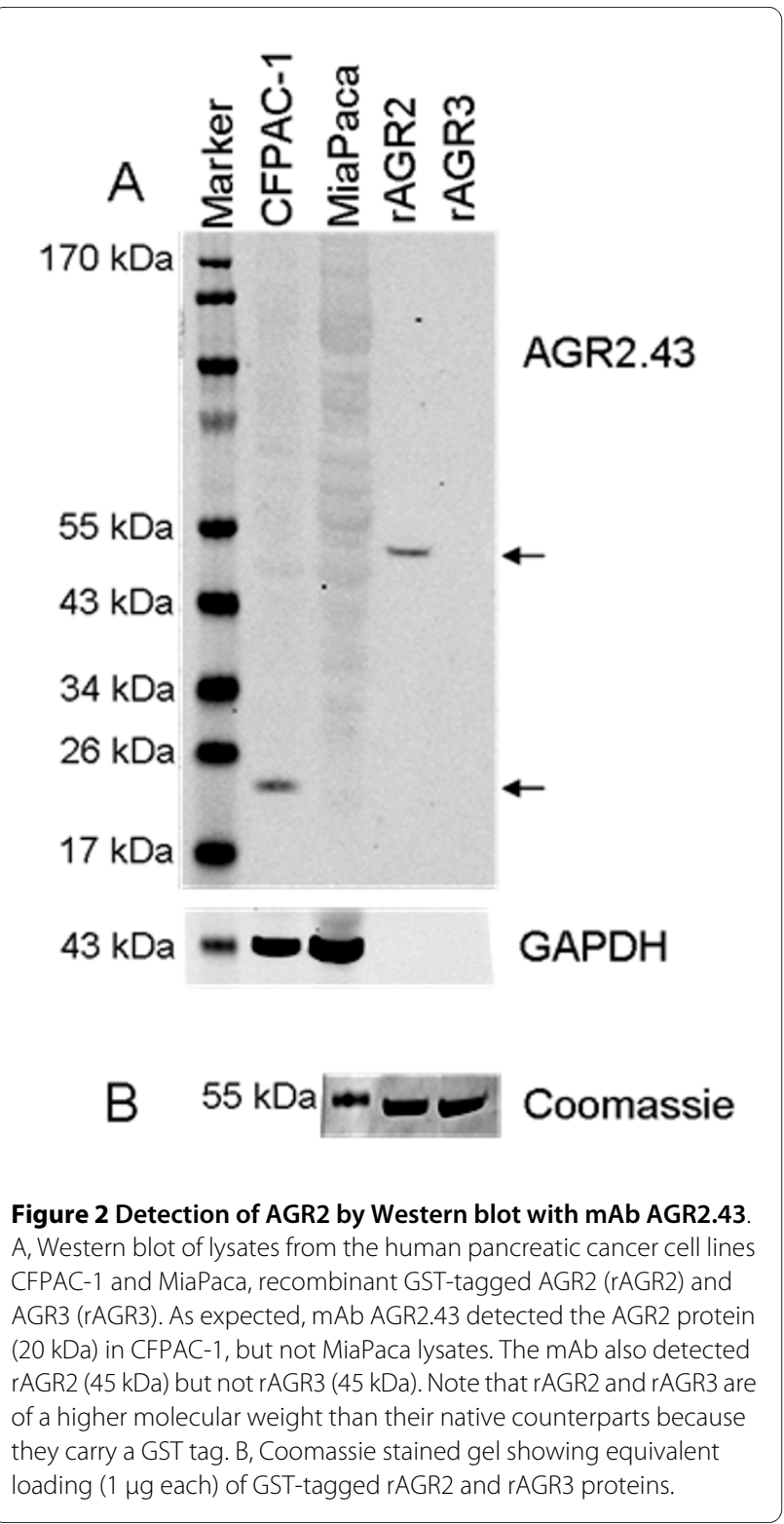

the expected AGR2 band. mAb AGR2.43 was then used to immunoprecipitate proteins from the cell line OV90, and the immunoprecipitated proteins were analyzed by mass spectrometry. This revealed ten unique peptides covering $43 \%$ of the AGR2 protein; no peptides from the related protein AGR3 were detected (Table 3), suggesting no cross reactivity to AGR3. By ELISA, we could detect rAGR2 at concentrations as low as $0.055 \mathrm{ng} / \mathrm{ml}$ (Figure 3 and Table 4). In contrast, we failed to detect rAGR3 at concentrations of 4, 20, 100 or $500 \mathrm{ng} / \mathrm{ml}$ (data not shown). As expected, the ELISA detected higher levels of AGR2 protein in lysates from CFPAC-1 cells (120.7 ng/ $\mathrm{ml}$ ) compared to MiaPaca cells (14.6 ng/ml) (Table 4). By IHC, mAb AGR2.43 reacted with tissue sections from human PanINs and pancreatic cancer cases but not nor-

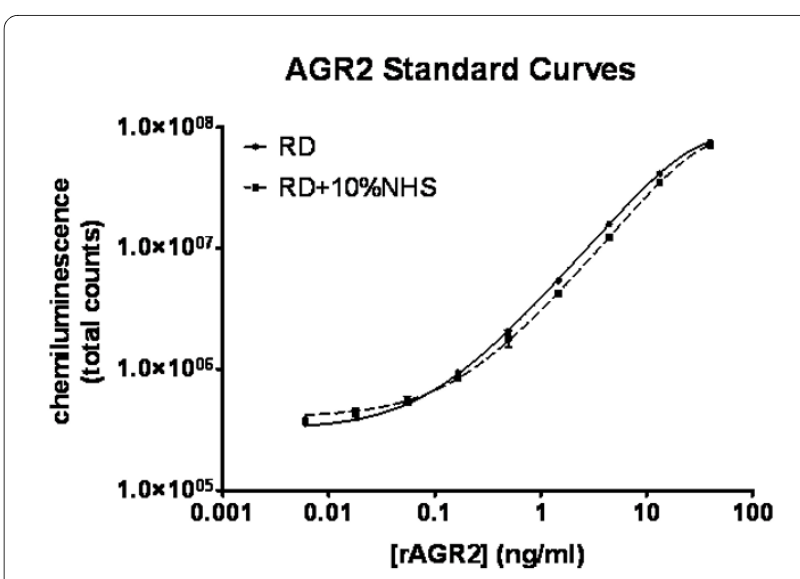

Figure 3 AGR2 ELISA standard curves. rAGR2 was diluted in either $1 \times$ Reagent Diluent (RD) or RD supplemented with 10\% normal human serum (RD+10\%NHS). A 3-fold dilution series of rAGR2 (from $40 \mathrm{ng} / \mathrm{ml}$ to $0.006 \mathrm{ng} / \mathrm{ml}$ ) was generated in RD or RD+NHS. AGR2 ELISA was used to measure the signal for each sample in $\mathrm{RD}$ (closed circles) or $\mathrm{RD}+10 \% \mathrm{NHS}$ (closed squares). Standard curves were generated for AGR2 in RD (solid line) or RD+10\%NHS (dashed line) using the five-parameter log-logistic method ( $r^{2}=0.999$ for both curves). The average of three replicates is presented. Error bars represent one standard deviation and are almost not apparent due to low intra-assay standard deviations.

mal pancreatic epithelium (Figure 4B). Taken together, these data confirm that mAb AGR2.43 is specific to AGR2 and can be used for multiple applications.

\section{Performance characteristics of the AGR2 ELISA}

We evaluated the AGR2 ELISA in different sample matrices using rAGR2, lysates from CFPAC-1 and MiaPaca cell lines, and a human serum sample. A 5PL standard curve was generated for each experiment and used to back-calculate the concentration of the rAGR2 standards and calculate AGR2 levels in lysates and serum. Figure 3 shows a standard curve of rAGR2 ranging from $0.006 \mathrm{ng} / \mathrm{ml}$ to 40 $\mathrm{ng} / \mathrm{ml}$. The curve produced an $\mathrm{r}$-squared of 0.999 demonstrating a good fit to the 5PL curve. The limit of detection, calculated as the mean value for the blank controls plus two standard deviations was $0.024 \mathrm{ng} / \mathrm{ml}$. The average signal to noise $(\mathrm{S}: \mathrm{N})$ ratios calculated from three separate experiments ranged from 3559 (40 $\mathrm{ng} / \mathrm{ml}$ standard) to $6.2(0.055 \mathrm{ng} / \mathrm{ml}$ standard $)$ suggesting a limit of detection of at least $0.055 \mathrm{ng} / \mathrm{ml} \mathrm{AGR2} \mathrm{(Table} \mathrm{4).} \mathrm{The} \mathrm{intra-}$ and inter-assay SD were calculated, and CVs ranged from $1.5 \%-5.9 \%$ and $1.6 \%-9.1 \%$, respectively. CFPAC-1 and MiaPaca lysates and the human serum sample were determined to have $120.7 \mathrm{ng} / \mathrm{ml}, 14.6 \mathrm{ng} / \mathrm{ml}$ and $22.0 \mathrm{ng} /$ ml AGR2 with intra-assay CVs of $6.8 \%, 5.4 \%$ and $6.2 \%$ respectively and inter-assay CVs of $4.3 \%, 6.8 \%$ and $16 \%$ respectively (Table 4). To assess the effects of serum on the AGR2 ELISA, we added 10\% Normal Human Serum (NHS) to a subset of samples. The resulting standard 


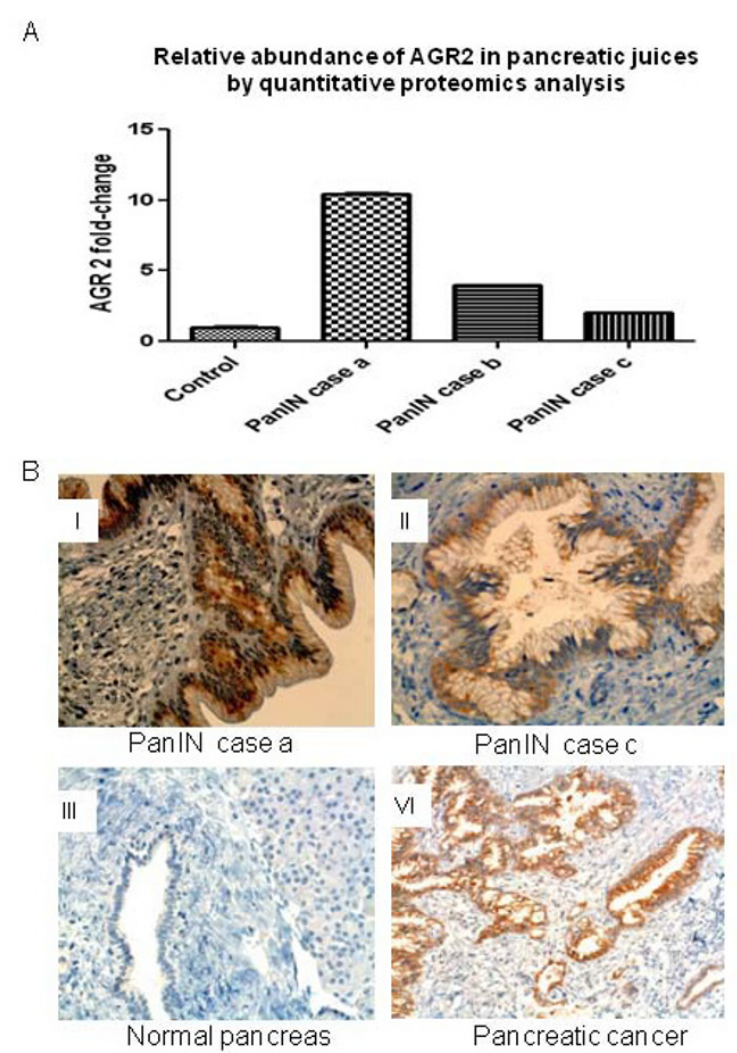

Figure 4 Elevation of AGR2. A. Fold-changes of AGR2 in pancreatic juice from patients with PanIN3 detected by quantitative proteomics analysis in comparison to controls. B. Detection of AGR2 by IHC in neoplastic pancreas ducts. Strong staining of AGR2 was detection in the neoplastic ducts of PanIN cases a and C (I and II), as well as the neoplastic region of pancreatic cancer (VI). AGR2 was not detected in the normal pancreas duct (III). curve produced an r-squared of 0.999 and closely mirrored the standard curve determined without serum (Figure 3).

\section{Correlation of AGR2 levels in pancreatic juice with its overexpression in neoplastic pancreas tissue}

We next examined AGR2 expression by IHC in pancreatic tissues corresponding to the pancreatic juice samples from cases a and $\mathrm{c}$ in the above quantitative proteomics analysis. As shown in Figure 4B, AGR2 was highly expressed in the PanIN ducts from both PanIN cases a and c, and also highly expressed (strong staining) in pancreatic cancer, but not in normal pancreas epithelium (negative staining). Thus, the elevation of AGR2 level in the pancreatic juice was correlated with high expression in PanIN ducts. Previous study had reported high expression of AGR2 in neoplastic cells with 98\% (56 of 57) positivity on pancreatic cancer and minimal staining in normal and pancreatitis tissues [11]. Our current study suggests that the detected AGR2 in pancreatic juices is likely originated from the pancreas.

\section{Significant elevation of AGR2 levels in the pancreatic juice samples from patients with pre-malignant pancreatic neoplasia}

We used ELISA to measure AGR2 levels in pancreatic juice samples from patients with benign pancreatic diseases ( $\mathrm{n}=18$; including chronic pancreatitis), premalignant pancreatic neoplasia $(\mathrm{n}=25$; including PanIN2, PanIN3, and IPMNs), and pancreatic cancer $(\mathrm{n}=8)$. As shown in Figure 5A and B, the level of AGR2 in pancreatic juice was significantly higher in patients with premalignant pancreatic diseases compared to benign disease controls $(p=0.003)$. The level of pancreatic juice

Table 3: Peptides identified from the AGR2 pull-down product

\begin{tabular}{lccc}
\hline AGR2 Peptide Observed & Sequence Position & Mass detected & $\begin{array}{c}\text { PeptideProphet } \\
\text { probability score }\end{array}$ \\
& & & 0.93 \\
KDTKDSRPKLPQTLSR & $51-66$ & 1869.04 & 1.00 \\
DSRPKLPQTLSR & $55-66$ & 1396.78 & 0.92 \\
LPQTLSR & $60-66$ & 813.47 & 1.00 \\
GWGDQLIWTQTYEEALYK & $67-84$ & 2200.05 & 1.00 \\
GWGDQLIWTQTYEEALYKSK & $67-86$ & 2415.17 & 1.00 \\
LAEQFVLLNLVYETTDKHLSPDGQYVPR & $120-147$ & 3244.68 & 1.00 \\
NLVYETTDKHLSPDGQYVPR & $128-147$ & 2331.15 & 1.00 \\
IMFVDPSLTVR & $148-158$ & 1276.68 & 1.00 \\
IML147]FVDPSLTVR & $148-158$ & 1293.83 & 0.99 \\
ALKLLKTEL & $187-195$ & 1027.66 & \\
\hline
\end{tabular}

Note: [147] indicates oxidized methionine 
Table 4: AGR2 ELISA performance characteristics

\begin{tabular}{|c|c|c|c|c|c|c|c|c|c|c|c|}
\hline & & \multicolumn{7}{|c|}{ Recombinant AGR2 Protein (ng/ml) } & \multirow[t]{2}{*}{ Human Serum } & \multirow{2}{*}{$\begin{array}{c}\text { Panc. Cell Lysates } \\
\text { CFPAC1-1 }\end{array}$} & \multirow[b]{2}{*}{ MiaPaca } \\
\hline & & 40 & 13.3 & 4.44 & 1.48 & 0.494 & 0.165 & 0.055 & & & \\
\hline \multirow[t]{2}{*}{ All Assays } & Mean (ng/ml) & 40.30 & 13.20 & 4.54 & 1.41 & 0.48 & 0.18 & 0.07 & 22.00 & 120.70 & 14.60 \\
\hline & ratio (signal/noise) & 3559 & 1169 & 401 & 124 & 42 & 15 & 6.2 & 195 & 1065 & 129 \\
\hline \multirow[t]{2}{*}{ Intra-assay } & $\mathrm{SD}(\mathrm{ng} / \mathrm{ml})$ & 1.260 & 0.199 & 0.079 & 0.062 & 0.014 & 0.008 & 0.004 & 1.400 & 8.300 & 0.790 \\
\hline & CV (\%) & 3.1 & 1.5 & 1.8 & 4.4 & 2.9 & 4.6 & 5.9 & 6.2 & 6.8 & 5.4 \\
\hline \multirow[t]{2}{*}{ Inter-assay } & $\mathrm{SD}(\mathrm{ng} / \mathrm{ml})$ & 1.240 & 0.207 & 0.081 & 0.067 & 0.012 & 0.008 & 0.006 & 3.400 & 5.100 & 1.000 \\
\hline & CV (\%) & 3.1 & 1.6 & 1.8 & 4.8 & 2.6 & 4.5 & 9.1 & 16.0 & 4.3 & 6.8 \\
\hline
\end{tabular}

SD: standard deviation; CV: coefficient of variation

AGR2 was also significantly higher in pancreatic cancer compared to controls $(p=0.03)$. The median pancreatic juice AGR2 level was not significantly different between males and females, different age groups, within subcategories of benign controls and premalignant pancreatic diseases (all p $>0.05$, Table 5).

ROC analysis was applied to evaluate the sensitivity and specificity of AGR2 in distinguishing premalignant and pancreatic cancer juice samples from benign controls (Figure 6). At 90\% specificity, the AGR2 ELISA achieved $40 \%$ sensitivity in discriminating premalignant juice samples from the control group (Table 6). The area under curve (AUC) was 0.742 . When we compared the ROC curve of the subset of PanIN3 juice samples to the control group, the AUC was slightly improved to 0.765 . At $90 \%$ specificity, the AGR2 ELISA achieved $67 \%$ sensitivity in discriminating PanIN3 juice samples from the control group. Pancreatic cancer juice samples achieved a slightly lower AUC value (AUC = 0.729) and sensitivity than the premalignant group (Table 6). At 90\% specificity, AGR2 had a sensitivity of $25 \%$ in discriminating pancreatic cancer juice samples from the control group.

\section{Non-correlation of AGR2 levels in pancreatic juice versus} serum

AGR2 levels were measured by ELISA in six paired serum and juice samples (all from PanIN patients). AGR2 was not detectable in any of the six serum samples tested. Of the corresponding juice samples, five displayed positive AGR2 levels, ranging from $0.84 \mathrm{ng} / \mathrm{ml}$ to $17.91 \mathrm{ng} / \mathrm{ml}$. Thus, AGR2 levels in pancreatic juice do not correlate with AGR2 levels in serum. Moreover, when we com-
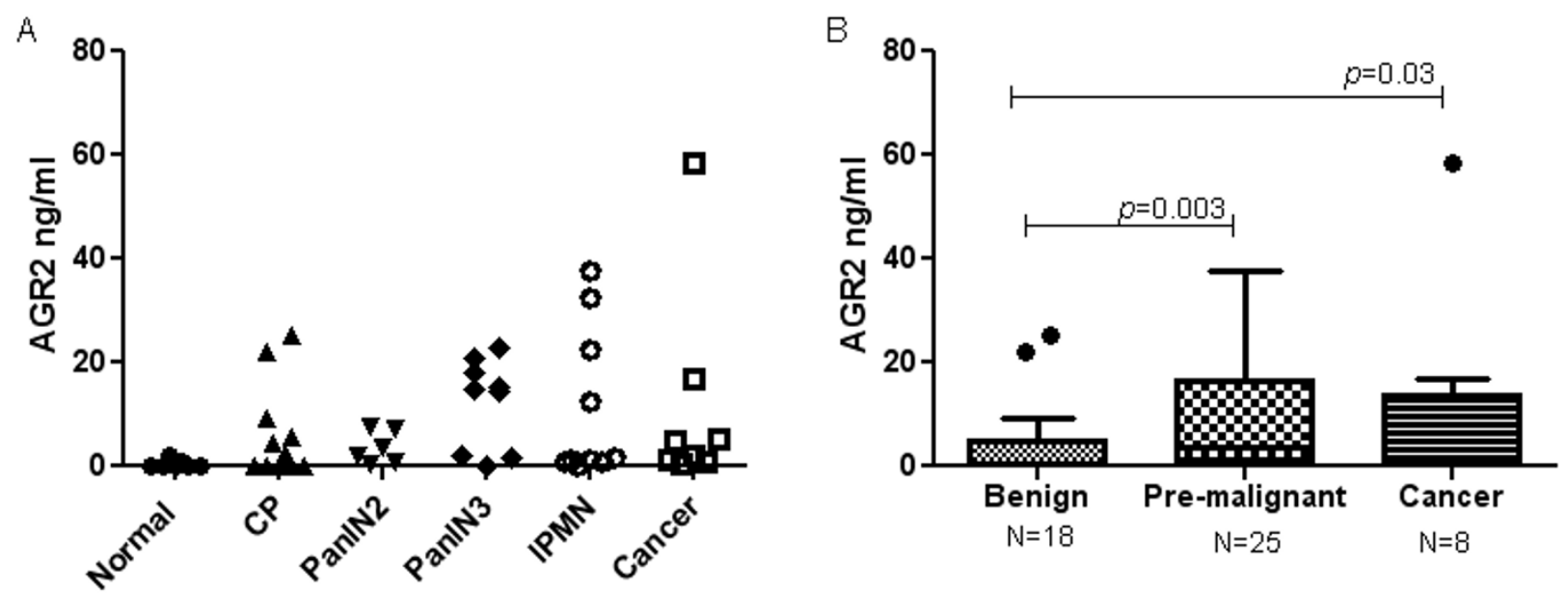

Figure 5 A. AGR2 concentration in pancreatic juice as measured by ELISA. CP: chronic pancreatitis. B. Boxplots of AGR2 concentration in pancreatic juices from patients with benign diseases ( $n=20$, including normal and chronic pancreatitis), pre-malignant lesions ( $n=25$, including PanIN2, PanIN3 and IMPNs), and pancreatic cancer $(n=8)$. Boxplot: the thin box marks out the 25th to 75 th percentiles; the line marks the outer part of the distribution, and outside dots mark outliers. 
Table 5: Median pancreatic juice AGR2 values by clinical-pathologic parameters

\begin{tabular}{|c|c|c|c|}
\hline & number of case & $\begin{array}{l}\text { Juice Median } \\
\text { AGR2 (ng/ml) }\end{array}$ & $P$ \\
\hline Sex & & & 0.35 \\
\hline Male & 19 & 1.82 & \\
\hline Female & 30 & 1.94 & \\
\hline Age & & & 0.79 \\
\hline$Y(<50)$ & 24 & 2.09 & \\
\hline$M(50-70)$ & 17 & 1.82 & \\
\hline $\mathrm{O}(>70)$ & 10 & 3.61 & \\
\hline Benign & & & 0.12 \\
\hline Sphincter of Oddi & 7 & 0.00 & \\
\hline Chronic pancreatitis & 11 & 2.32 & \\
\hline Premalignant & & & 0.36 \\
\hline PanIN2 & 6 & 2.88 & \\
\hline PanIN3 & 9 & 14.85 & \\
\hline IPMN & 10 & 1.49 & \\
\hline Malignant & 8 & 4.93 & \\
\hline
\end{tabular}

pared sera from pancreatic cancer cases and cancer-free controls, only $1 / 9$ pancreatic cancer sera showed a high level of AGR2, compared to 0/9 cancer-free controls. Therefore, AGR2 levels were not significantly different between pancreatic cancer sera and sera from cancer-free controls (Figure 7, $p=0.27$ ).

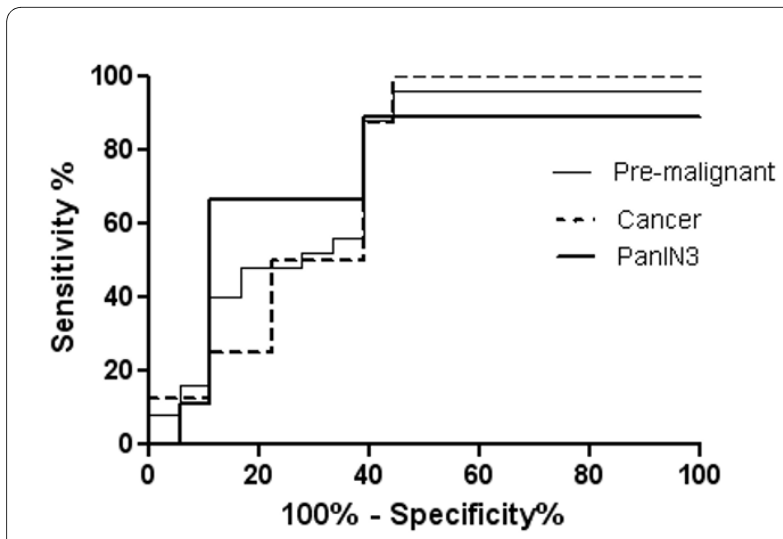

Figure 6 ROC curves of AGR2 levels in pancreatic juice from patients with pre-malignant lesions, PanIN3 lesions, and pancreatic cancer compared to benign disease controls.

\section{Discussion}

AGR2 is a secreted protein initially described in Xenopus laevis for being differentially expressed in neural development [19]. In Xenopus, the AGR2 homologue is critical in forming anterior structures during embryonic ectoderm development [20]. The function of AGR2 in human tissue is largely unknown, but overexpression of AGR2 has been reported in several cancers, including breast, prostate, lung and pancreas and in circulating tumor cells [11,2125]. Recent evidence suggests that AGR2 can promote tumor growth, promote cancer cell survival, cell migration, and cellular transformation [11,19]. Detection of AGR2 in these previous studies was primarily performed on tissue or cell lines using RT-PCR, western blotting or IHC. One recent study showed that increased AGR2 in plasma is associated with ovarian cancer[26]. However, to the best of our knowledge, evaluation of AGR2 protein in plasma or other biological fluids has not been reported in pancreatic cancer.

In this study, we first used quantitative proteomics to identify elevated proteins in pancreatic juice. We found that AGR2 was elevated in all of the three pre-neoplastic pancreatic juice samples (PanIN3) analyzed by quantitative proteomics. The AGR2 levels in pancreatic juice correlated with its over-expression in matching pancreas 
Table 6: Pancreatic juice AGR2 ROC analysis

\begin{tabular}{lccc}
\hline & Premalignant vs control & PanIN3 vs control & Cancer vs control \\
\hline Area under curve (AUC) & 0.742 & 0.765 & 0.729 \\
Sensitivity at 95\% specificity & $16 \%$ & $11 \%$ & $11 \%$ \\
Sensitivity at 90\% specificity & $40 \%$ & $67 \%$ & $25 \%$ \\
\hline
\end{tabular}

tissues. These findings are in concert with previous studies that have also demonstrated increased expression of AGR2 in PanIN lesions [11,27] and pancreatic ductal adenocarcinoma tissues $[11,23]$. Along with these previous studies, it is evidenced that AGR2 is likely to be secreted into the pancreatic duct fluid early in pancreatic cancer progression. However, a recent study found that AGR2 is localized to the endoplasmic reticulum in intestinal epithelial cells and did not detect AGR2 in secretory granules or in the intestinal lumen[28]. Thus it is possible that only a fraction of AGR2 is actually secreted. And this could explain why pancreatic cancer patients do not have higher juice AGR2 level than the PanIN patients, while they have strong AGR2 staining in the cancer tissues. The mechanism governing AGR2 secretion is currently unknown.

To evaluate AGR2 levels in pancreatic juice and blood, we developed an ELISA assay to quantitatively measure AGR2 levels. We used mass spectrometry to verify that the monoclonal antibody used in the ELISA was indeed specific for AGR2. By ELISA, AGR2 levels in the pancreatic juice samples from patients with benign pancreatic disease, premalignant pancreatic neoplasia and pancre-

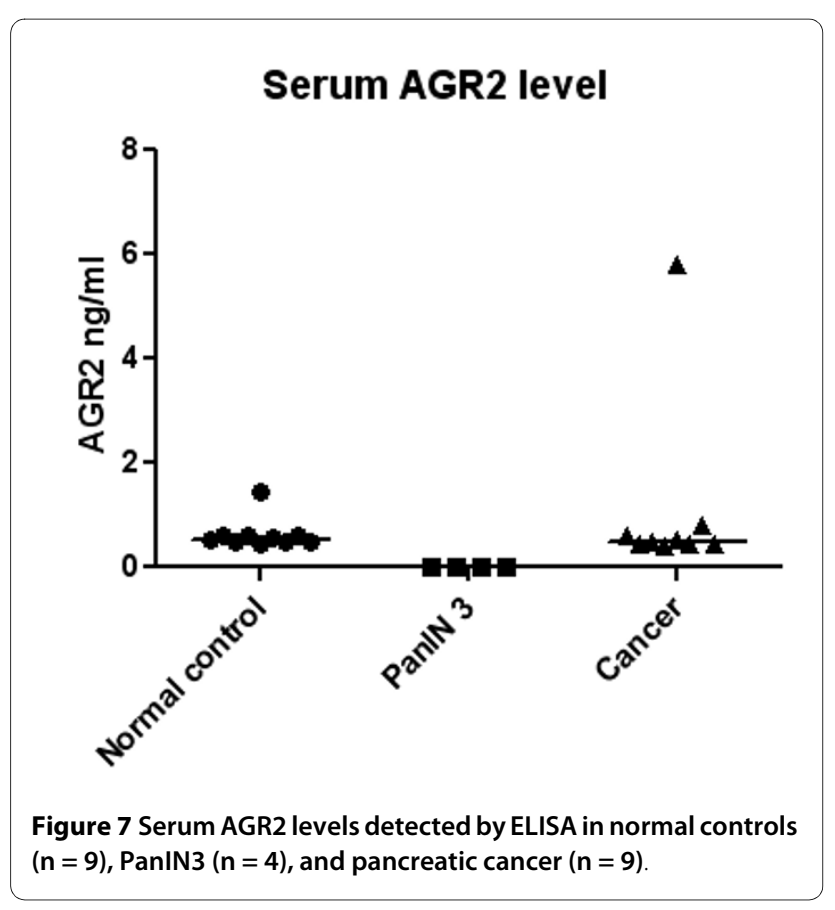

atic cancer were tested and compared. The pancreatic juice AGR2 level was significantly elevated in patients with premalignant lesions and pancreatic cancer compared to control samples. Further ROC analysis suggested that at $90 \%$ specificity, the AGR2 ELISA achieved 67\% sensitivity in detecting PanIN3 juice samples from the control group. The ability to detect pancreatic cancer at an early stage could greatly benefit the patient management and improve the survival rate. Currently, it is only possible to detect PanIN3 histologically. Pancreatic juice CA19-9 levels could not separate patients with pancreatic cancer from chronic pancreatitis[29]. Moreover, CA19-9 is not elevated in the blood of PanIN3 patients, thus is not useful as a PanIN3 biomarker[12]. The discovery of AGR2 elevation in the pancreatic juice of PanIN3 patients in this study may provide a future opportunity to develop and test this candidate as juice biomarker for PanIN3.

While AGR2 levels were significantly elevated in the pancreatic juice from premalignant and pancreatic cancer patients, serum levels of AGR2 did not distinguish patients with premalignant or pancreatic cancer from control patients. This result supports the view that pancreatic juice, due to proximity to the tumor, can be a more sensitive and specific source for identifying biomarker candidates associated with pancreatic cancer or pre-cancer $[30,31]$.

\section{Conclusion}

In this study, we found that AGR2 was significantly elevated in the pancreatic juice from patients with premalignant conditions (PanINs and IPMNs) as well as pancreatic cancer compared to control pancreatic juice samples. AGR2 levels in pancreatic juice could potentially be used to aide in assessment of high-risk patients undergoing endoscopic procedures.

Competing interests

The authors declare that they have no competing interests.

\section{Authors' contributions}

$\mathrm{RC}$ and SP carried out the quantitative proteomics study. RC, SP, and XD drafted the manuscript. XD, BHN, RAS, SR generated monoclonal antibodies and carried out the immunoassays. RC, SP, BHN, RAK, MM and TAB participated in the design of the study and analysis of the data. All authors commented, read and approved the final manuscript.

\section{Acknowledgements}

This work was supported by K07CA116296, R01CA107209, K25CA137222, R01DK081368, and the AACR-PanCAN Career Development Award for Pancre- 
atic Cancer Research, with additional funding from the Canary Foundation, Gene and Mary Ann Walters Pancreatic Cancer Foundation, Lustgarten Foundation, and British Columbia Cancer Foundation. We thank the Proteomics Facility at the Institute for Systems Biology for mass spectrometric analysis.

\section{Author Details}

'GI Division/Department of Medicine, University of Washington, Seattle, WA 98195 USA, 2Department of Pathology, University of Washington, Seattle, WA 98195 USA, 3BC Cancer Agency, Victoria, BC, V8R6V5 Canada, 4Virginia Mason Medical center, Seattle, WA 98101 USA and ${ }^{5}$ Division of Public Health, Fred Hutchinson Cancer Research Center, Seattle, WA 98109 USA

Received: 19 November 2009 Accepted: 15 June 2010 Published: 15 June 2010

\section{References}

1. Jemal A, Thomas A, Murray T, Thun M: Cancer statistics, 2002. CA Cancer $J$ Clin 2002, 52:23-47.

2. Goggins M: Identifying molecular markers for the early detection of pancreatic neoplasia. Semin Oncol 2007, 34:303-310.

3. Grote T, Logsdon CD: Progress on molecular markers of pancreatic cancer. Curr Opin Gastroenterol 2007, 23:508-514.

4. Habbe N, Koorstra JB, Mendell JT, Offerhaus GJ, Ryu JK, Feldmann G, Mullendore ME, Goggins MG, Hong SM, Maitra A: MicroRNA miR-155 is a biomarker of early pancreatic neoplasia. Cancer Biol Ther 2009, 8:340-346.

5. Matsubayashi H, Canto M, Sato N, Klein A, Abe T, Yamashita K, Yeo CJ, Kalloo A, Hruban R, Goggins M: DNA methylation alterations in the pancreatic juice of patients with suspected pancreatic disease. Cancer Res 2006, 66:1208-1217.

6. Misek DE, Patwa TH, Lubman DM, Simeone DM: Early detection and biomarkers in pancreatic cancer. J Natl Compr Canc Netw 2007, 5:1034-1041.

7. Ohuchida K, Mizumoto K, Yamada D, Yamaguchi H, Konomi H, Nagai E, Yamaguchi K, Tsuneyoshi M, Tanaka M: Quantitative analysis of human telomerase reverse transcriptase in pancreatic cancer. Clin Cancer Res 2006, 12:2066-2069.

8. Ohuchida K, Mizumoto K, Yamada D, Fujii K, Ishikawa N, Konomi H, Naga E, Yamaguchi K, Tsuneyoshi M, Tanaka M: Quantitative analysis of MUC and MUC5AC mRNA in pancreatic juice for preoperative diagnosis of pancreatic cancer. Int J Cancer 2006, 118:405-411.

9. Chen R, Pan S, Aebersold R, Brentnall TA: Proteomics studies of pancreatic cancer. Proteomics Clin Appl 2007, 1:1582-1591.

10. Harsha HC, Kandasamy K, Ranganathan P, Rani S, Ramabadran S, Gollapudi S, Balakrishnan L, Dwivedi SB, Telikicherla D, Selvan LD, Goel R, Mathivanan S, Marimuthu A, Kashyap M, Vizza RF, Mayer RJ, Decaprio JA, Srivastava S, Hanash SM, Hruban RH, Pandey A: A compendium of potential biomarkers of pancreatic cancer. PLoS Med 2009, 6:e1000046.

11. Ramachandran V, Arumugam T, Wang H, Logsdon CD: Anterior gradient 2 is expressed and secreted during the development of pancreatic cancer and promotes cancer cell survival. Cancer Res 2008, 68:7811-7818

12. Brentnall TA, Bronner MP, Byrd DR, Haggitt RC, Kimmey MB: Early diagnosis and treatment of pancreatic dysplasia in patients with a family history of pancreatic cancer. Ann Intern Med 1999, 131:247-255.

13. Ross PL, Huang YN, Marchese JN, Williamson B, Parker K, Hattan S, Khainovski N, Pillai S, Dey S, Daniels S, Purkayastha S, Juhasz P, Martin S, Bartlet-Jones M, He F, Jacobson A, Pappin DJ: Multiplexed protein quantitation in Saccharomyces cerevisiae using amine-reactive isobaric tagging reagents. Mol Cell Proteomics 2004, 3:1 154-1169.

14. Pan S, Chen R, Reimel BA, Crispin DA, Mirzaei H, Cooke K, Coleman JF, Lane Z, Bronner MP, Goodlett DR, McIntosh MW, Traverso W, Aebersold R, Brentnall TA: Quantitative proteomics investigation of pancreatic intraepithelial neoplasia. Electrophoresis 2009, 30:1132-1144.

15. Eng J, McCormack AL, Yates JR: An approach to correlate tandem mass spectral data of peptides with amino acid sequences in a protein database. J Am Soc Mass Spectrom 1994, 5:976-989.

16. Keller A, Nesvizhskii Al, Kolker E, Aebersold R: Empirical statistical model to estimate the accuracy of peptide identifications made by MS/MS and database search. Analytical Chemistry 2002, 74:5383-5392.
17. Nesvizhskii Al, Keller A, Kolker E, Aebersold R: A statistical model for identifying proteins by tandem mass spectrometry. Anal Chem 2003, 75:4646-4658

18. Deutsch EW, Lam H, Aebersold R: PeptideAtlas: a resource for target selection for emerging targeted proteomics workflows. EMBO Rep 2008, 9:429-434.

19. Wang Z, Hao Y, Lowe AW: The adenocarcinoma-associated antigen, AGR2, promotes tumor growth, cell migration, and cellular transformation. Cancer Res 2008, 68:492-497.

20. Aberger F, Weidinger G, Grunz H, Richter K: Anterior specification of embryonic ectoderm: the role of the Xenopus cement gland-specific gene XAG-2. Mech Dev 1998, 72:115-130.

21. Fritzsche FR, Dahl E, Pahl S, Burkhardt M, Luo J, Mayordomo E, Gansukh T, Dankof A, Knuechel R, Denkert C, Winzer KJ, Dietel M, Kristiansen G: Prognostic relevance of AGR2 expression in breast cancer. Clin Cancer Res 2006, 12:1728-1734.

22. Fritzsche FR, Dahl E, Dankof A, Burkhardt M, Pahl S, Petersen I, Dietel M, Kristiansen G: Expression of AGR2 in non small cell lung cancer. Histol Histopathol 2007, 22:703-708.

23. Riener MO, Pilarsky C, Gerhardt J, Grutzmann R, Fritzsche FR, Bahra M, Weichert W, Kristiansen G: Prognostic significance of AGR2 in pancreatic ductal adenocarcinoma. Histol Histopathol 2009, 24:1121-1128.

24. Zhang Y, Forootan SS, Liu D, Barraclough R, Foster CS, Rudland PS, Ke Y: Increased expression of anterior gradient-2 is significantly associated with poor survival of prostate cancer patients. Prostate Cancer Prostatic Dis 2007, 10:293-300.

25. Valladares-Ayerbes M, Diaz-Prado S, Reboredo M, Medina V, Iglesias-Diaz P, Lorenzo-Patino MJ, Campelo RG, Haz M, Santamarina I, Anton-Aparicio LM: Bioinformatics approach to mRNA markers discovery for detection of circulating tumor cells in patients with gastrointestinal cancer. Cancer Detect Prev 2008, 32:236-250.

26. Edgell TA, Barraclough DL, Rajic A, Dhulia J, Lewis KJ, Armes JE, Barraclough R, Rudland PS, Rice GE, Autelitano DJ: Increased plasma concentrations of anterior gradient 2 protein are positively associated with ovarian cancer. Clin Sci (Lond) 2010, 118:717-725

27. Buchholz M, Braun M, Heidenblut A, Kestler HA, Kloppel G, Schmiegel W, Hahn SA, Luttges J, Gress TM: Transcriptome analysis of microdissected pancreatic intraepithelial neoplastic lesions. Oncogene 2005, 24:6626-6636

28. Park SW, Zhen G, Verhaeghe C, Nakagami Y, Nguyenvu LT, Barczak AJ, Killeen N, Erle DJ: The protein disulfide isomerase AGR2 is essential for production of intestinal mucus. Proc Natl Acad Sci USA 2009, 106:6950-6955.

29. Matsumoto S, Harada H, Tanaka J, Ochi K, Seno T, Tsurumi T, Kunichika K: Evaluation of cytology and tumor markers of pure pancreatic juice for the diagnosis of pancreatic cancer at early stages. Pancreas 1994 9:741-747.

30. Chen R, Pan S, Brentnall TA, Aebersold R: Proteomic profiling of pancreatic cancer for biomarker discovery. Mol Cell Proteomics 2005, 4:523-533.

31. Rosty C, Christa L, Kuzdzal S, Baldwin WM, Zahurak ML, Carnot F, Chan DW, Canto M, Lillemoe KD, Cameron JL, Yeo CJ, Hruban RH, Goggins M: Identification of hepatocarcinoma-intestine-pancreas/pancreatitisassociated protein I as a biomarker for pancreatic ductal adenocarcinoma by protein biochip technology. Cancer Res 2002, 62:1868-1875

doi: 10.1186/1476-4598-9-149

Cite this article as: Chen et al., Elevated level of anterior gradient- 2 in pancreatic juice from patients with pre-malignant pancreatic neoplasia Molecular Cancer 2010, 9:149 